\title{
Induction of Disease Resistance in Tea (Camellia sinensis L.) by Plant Activators
}

\author{
Katsuyuki YOSHIDA ${ }^{1 *}$, Akiko OGINO ${ }^{2}$, Kengo YAMADA ${ }^{3}$ and \\ Ryoichi SONODA ${ }^{3}$ \\ ${ }^{1}$ Tea Pest Management Research Team, National Institute of Vegetable and Tea Science, National \\ Agriculture and Food Research Organization (NARO) (Makurazaki, Kagoshima 898-0087, Japan) \\ ${ }^{2}$ Vegetable and Tea Function Research Team, National Institute of Vegetable and Tea Science, \\ NARO (Makurazaki, Kagoshima 898-0087, Japan) \\ ${ }^{3}$ Tea Pest Management Research Team, National Institute of Vegetable and Tea Science, NARO \\ (Shimada, Shizuoka 428-8501, Japan)
}

\begin{abstract}
About $75 \%$ of the tea fields in Japan grow cv. 'Yabukita,' which is susceptible to many diseases such as anthracnose and gray blight. Therefore, broad range effective and long-lasting disease control methods are required. Plant activators (PAs) are compounds that induce disease resistance in plants. In the present study, we used AGREVO EX (a yeast extract preparation), probenazole, prohydrojasmon, and tiadinil as PAs and estimated their disease resistance-inducing activity on tea plants by wound-inoculation assays and field trials. An aqueous solution of each PA was sprayed on fieldgrown 'Yabukita'. PA-treated leaves were harvested and assayed for disease resistance-inducing activity. Lesion development of gray blight and anthracnose in PA-treated leaves was suppressed by each PA, and the induced resistance continued for at least 30 days after PA treatment. All the tested PAs induced disease resistance in tea plants systemically. In field trials, PAs were effective to the diseases of anthracnose and gray blight with control efficacies from 50.1 to 50.8 and 63.7 to 69.8 respectively. The growth and chemical composition of tea shoots were not changed by PA treatment. Based on these experiments, induced resistance by PAs is useful for the development of new disease control methods for tea production.
\end{abstract}

Discipline: Plant disease / Plant protection

Additional key words: AGREVO EX, probenazole, prohydrojasmon, tianidil

\section{Introduction}

Tea [Camellia sinensis (L.) O. Kuntze] is one of the most important cash crops in the warm southwest areas of Japan. The green tea cultivar 'Yabukita' has been cultivated since the 1970 s and grown in about $75 \%$ of all tea fields in Japan. Using a monoculture cultivation system, new Yabukita tea shoots can be harvested synchronously, producing green tea of consistent quality. However, this system has resulted in severe pest problems such as outbreaks of tea anthracnose caused by Colletotrichum theae-sinensis (Miyake) Yamamoto ${ }^{32}$ and tea gray blight caused by Pestalotiopsis longiseta (Spegazzini) Dai et Kobayashi $^{22}$. Tea leaves are infected with C. theae-sinensis through trichomes at the tea shoot growing stage. Pestalotiopsis longiseta infects tea plants through the wound sites left after harvesting of tea shoots or cutting of the tea tree by tea-plucking machine. Therefore, repeated spraying of fungicides is needed to protect tea plants against both the diseases for each tea crop. In addition, the over-use of fungicide has caused the emergence of fungicide-resistant strains of C. theae-sinensis ${ }^{16}$ and $P$. longiseta ${ }^{17}$. In recent years, consumers have become more interested in food safety and like to buy chemical-free green tea. To reduce the use of chemicals in tea cultivation, it is important to develop new disease control methods that have a broad range and long-lasting disease control mechanisms without the emergence of fungicidal-resistant strains.

Plants do not have an immune system; however, they have developed unique defense mechanisms against the attack of microorganisms. Systemic acquired resistance (SAR) and induced systemic resistance (ISR) are

*Corresponding author: e-mail gohteny@affrc.go.jp

Received 28 September 2009; accepted 8 February 2010. 
the representative disease-resistance mechanisms in plants $^{1,4,18,20,24}$. SAR is induced by the activation of a salicylic acid (SA) signaling pathway in response to infection by parasitic pathogens ${ }^{1,4,18,20}$. ISR is caused by plant growth-promoting bacteria in the ethylene- and jasmonic acid (JA)-signaling pathway ${ }^{1,4,21,24}$. Therefore, SA, JA and ethylene are known as signal molecules of disease resistance against some pathogens in plants ${ }^{3,4,9,21,23-25}$. Plant activators (PAs) are chemical inducers of systemic and continuous disease resistance in plants without fungicidal

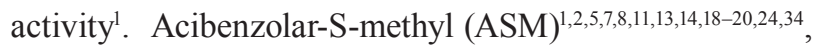
probenazole (PBZ, Oryzemate $\left.{ }^{\circledR}\right)^{1,18,33}$ and tinanidil (TDL, $\left.\mathrm{V}-\mathrm{Get}^{\circledR}\right)^{1,27,28}$ are PAs and PBZ has been used to control rice blast in Japan since 19741. PAs activate SAR, and therefore, they are expected to control a broad range of plant diseases without the emergence of fungicidal-resistant strains $\mathbf{s}^{1,18,24}$.

In the present study, we tried to use PAs for control of fungal diseases in tea plants. We tested the PA activity of PBZ, TDL, AGREVO EX (AGEX), and prohydrojasmon (PDJ) on tea plants. AGEX is a preparation of baker's yeast extract that induces disease resistance in tobacco through the synthesis of basic PR-proteins and ethylene $^{15}$. PDJ is a synthetic jasmonate derivative that has the same bioactivity as JA ${ }^{12}$. The mechanism of induced disease resistance in tea plants has not been analyzed. Therefore, we used AGEX, PBZ, PDJ, and TDL as PAs in this study because these substances have different action sites in the plant defense signaling pathway. We assayed the disease resistance-inducing activity of PAs on anthracnose and gray blight in tea plants by wound inoculation. We also tested the disease control efficacy of PAs on tea plants in a field trial and determined the effect of PAs on its growth and chemical composition.

\section{Materials and methods}

\section{Plant and pathogens}

The green tea cultivar 'Yabukita' was used in this study. Field grown and matured tea trees were used for wound-inoculation assays and field trials. Colletotrichum theae-sinensis (Cts, isolate SI-4-2) and P. longiseta (Pl, isolate NP2-2-1) were used in this study. These fungi were cultivated on autoclaved tea stems in an Erlenmeyer flask for a month at $26^{\circ} \mathrm{C}$ and a conidial suspension was prepared as described previously ${ }^{32}$.

\section{PA and chemicals}

AGEX was provided by AGREVO Co. Ltd. PBZ (as $48 \%$ active ingredient [a.i.] in a wettable powder formulation) and PDJ (as 5\% a.i. in a PDJ solution) were purchased from Meiji Seika Co. Ltd. TDL (as 30\% a.i. in a flowable formulation) was kindly donated by Nihon Nouyaku Co. Ltd. Chlorothalonil (TPN, as 40\% a. i. in a flowable formulation), a fungicide commonly used for the control of fungal diseases of tea in Japan, was purchased from Sumitomo Chemical Co. Ltd.

\section{Bioassay of PA activity}

For the analysis of PA activity in tea plants, aqueous solutions of each PA were sprayed on a mature first flush of field-grown tea plants. PA-treated tea lops with two mature leaves were harvested 5-30 days after PA treatments. Tea leaves were inoculated with $\mathrm{Cts}$ or Pl by wound inoculation just after harvest. The leaves were inoculated with Cts by a previously described method ${ }^{32}$, and with Pl by the method of Yanase and Takeda ${ }^{26}$ with some modifications. After inoculation, tea lops with two inoculated leaves were planted in a ROOTCUBES ${ }^{\circledR}$ growing medium (Smithers-Oasis Co.) and cultivated by the same method as used for the wound-inoculation assay of $\mathrm{Cts}^{32}$. The lesion sizes of each pathogen were measured 2 weeks after inoculation. To analyze the long-term induction of disease resistance by PAs, AGEX and TDL were sprayed on newly emerging shoots of a field-grown tea plant. Lops of PA-treated tea plants were harvested 42 days after PA treatment and inoculated with Pl, as described above. The systemic induction of disease resistance by PAs was also observed. Lops (about $10 \mathrm{~cm}$ length) with two mature leaves were collected from the field. After washing with tap water and surface drying, the lower leaf of the lop was sprayed with PAs using a TLC sprayer. The treated lops were cultivated for $48 \mathrm{~h}$ in a growth chamber $\left(26^{\circ} \mathrm{C}, 12 \mathrm{~h} / 12 \mathrm{~h}\right.$ light/dark, RH 90\%). An upper leaf (not treated with PA) of the lop was inoculated with $\mathrm{Pl}$, as described above, and the lesion size was estimated 2 weeks after inoculation.

\section{Assay of anti-fungal activity of PA}

The antifungal activities of PAs on $\mathrm{Cts}$ and $\mathrm{Pl}$ were determined by its inhibitory effect on mycelial growth. $\mathrm{Cts}$ and Pl were precultured on PDA (Merck) plates for 10 days. Mycelium disks (4 $\mathrm{mm}$ in diameter) of Cts or Pl were cut from the mycelial front of the PDA plate and inoculated on a PA-added Czapek's agar plate. Mycelial growth was measured 14 days after inoculation.

\section{Field trials}

To estimate the control efficacy of PAs on anthracnose, tea plants were sprayed with the aqueous solutions of PAs (200 L/10 a) at the tea shoot emerging stage of the third tea crop. Tea plants were inoculated with a conidial suspension of Cts $\left(70 \mathrm{~L} / 10 \mathrm{a}, 1 \times 10^{7}\right.$ conidia/mL) 3 days after PA treatment. The number of leaves infected with 
Cts was counted 4 weeks after inoculation. To estimate the control efficacy of PAs on gray blight, tea plants were inoculated with a conidial suspension of $\mathrm{Pl}(70 \mathrm{~L} / 10 \mathrm{a}, 1 \times$ $10^{5}$ conidia $/ \mathrm{mL}$ ) immediately after harvesting the tea shoots with a tea-plucking machine at the end of the second tea crop. Tea plants were sprayed with aqueous solutions of PAs (200 L/10 a) $4 \mathrm{~h}$ after inoculation. TPN was used as a positive control in field trials. The control efficacy of PAs was estimated by comparing the number of infected leaves of the control and PA-treated plot. The size of each experimental plot was $3 \times 1.8 \mathrm{~m}$ (length $\times$ width) of tea hedge and three experimental areas were prepared for each treatment. The number of infected leaves at a 50 -cm-square $\left(0.25 \mathrm{~m}^{2}\right)$ was counted at six points in each experimental area. Mean number of infected leaves per $1 \mathrm{~m}^{2}$ was calculated and statistically analyzed by Tukey's test. Control efficacy was determined according to the following equation: Control efficacy $=$ 100 - (Average number of infected leaves in an experimental plot/Average number of infected leaves at the control plot) $\times 100$.

\section{Effect of PA on growth and chemical composition of tea shoots}

Tea plants were sprayed with the aqueous solutions of PAs (200 L/10 a) at the tea shoot emerging stage of the third tea crop and tea shoots were harvested 3 weeks after PA treatment. The differences in the growth of tea shoots treated with each PA were determined by measuring the length, number of leaves and the weight of 40 tea shoots. About $30 \mathrm{~g}$ of tea shoots were dried and ground into a powder after the steaming treatment. The major chemical constituents of tea leaves were determined by Shi- zuokaseiki GT-8S, a near infrared spectroscope for the measurement of the major tea constituents associated with green tea quality. The contents of total nitrogen, total free amino acids, neutral detergent fiber, tannin, and caffeine were determined according to the manufacturer's instruction.

\section{Results}

\section{PA induces continuous and systemic disease resis- tance in tea plant}

Field grown tea plants were treated with four kinds of PA. Tea leaves were harvested and inoculated with Cts or Pl at 5, 10 and 30 days after PA treatment. In control leaves, $\mathrm{Pl}$ and $\mathrm{Cts}$ lesions were developed at each inoculation. Cts lesion developments were suppressed in PAtreated leaves (Fig. 1, Table 1). The lesion sizes in PAtreated leaves were less than $5 \mathrm{~mm}$ in all the experiments. There was no difference between any of the PAs in terms of inducible resistance to Cts lesion development. Suppression of Pl lesion size in PA-treated leaves was also observed (Fig. 1, Table 1). However, the Pl lesion size in PDJ-treated leaves was slightly larger than that of leaves treated with other PAs. PA application to emerging tea shoots also induced disease resistance in tea plants. P1 were inoculated to mature lop leaves which were sprayed with TDL or AGEX for 42 days before inoculation. Pl lesion size was significantly suppressed in TDL- and AGEX-treated leaves, but only slightly suppressed in TPN-treated leaves (Fig. 2). Pl lesion size was significantly suppressed in the upper leaves of PA-treated lops as compared to that in the control (Fig. 3). Suppression of Pl was the same by each PA treatment.
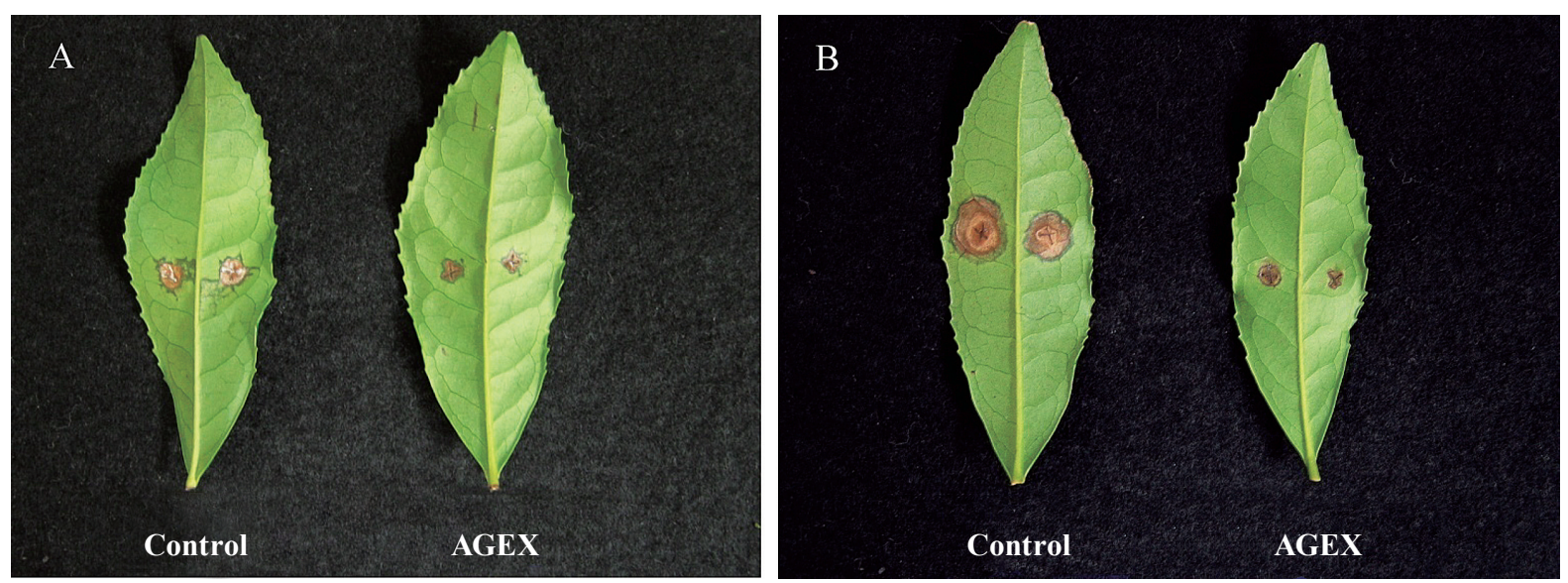

Fig. 1. Suppression of lesion developments of anthracnose (A) and gray blight (B) in 0.1\% AGREVO EX (AGEX) treated tea leaves

Colletotrichum theae-sinensis and P. longiseta were inoculated 10 days after AGEX treatment. These photos were taken 14 days after inoculation. 
Table 1. Suppression of lesion development of anthracnose and gray blight in plant activator-treated tea leaves

\begin{tabular}{|c|c|c|c|c|c|c|}
\hline \multirow{2}{*}{ Treatment } & \multicolumn{3}{|c|}{ Lesion size in anthracnose (mm) } & \multicolumn{3}{|c|}{ Lesion size in gray blight $(\mathrm{mm})$} \\
\hline & $5^{1)}$ & 10 & 30 & 5 & 10 & 30 \\
\hline Contol & $5.8 a^{2)}$ & $8.2 \mathrm{a}$ & $5.9 \mathrm{a}$ & $9.6 \mathrm{a}$ & $10.2 \mathrm{a}$ & $12.3 \mathrm{a}$ \\
\hline AGEX $0.1 \%$ & $3.6 \mathrm{~b}$ & $4.6 b$ & $4.6 \mathrm{~b}$ & $3.5 \mathrm{c}$ & $4.1 b$ & $5.3 b$ \\
\hline $\mathrm{PBZ} 240 \mu \mathrm{g} / \mathrm{mL}$ & $4.0 \mathrm{~b}$ & $4.9 \mathrm{~b}$ & $4.1 b$ & $5.8 \mathrm{~b}$ & $4.6 b$ & $6.6 \mathrm{~b}$ \\
\hline PDJ $100 \mu \mathrm{g} / \mathrm{mL}$ & $3.6 \mathrm{~b}$ & $4.8 b$ & $4.4 b$ & $5.5 b$ & $6.3 \mathrm{c}$ & $8.7 \mathrm{c}$ \\
\hline TDL $300 \mu \mathrm{g} / \mathrm{mL}$ & $3.7 b$ & $4.7 b$ & $4.1 \mathrm{~b}$ & $3.3 \mathrm{c}$ & $3.5 b$ & $5.5 b$ \\
\hline
\end{tabular}

1) Values indicate the number of inoculation days after plant activator treatment.

2) Lesion sizes were estimated 14 days after inoculation $(n=24)$. Different letters denote a significant difference at the $1 \%$ level by Tukey's test.

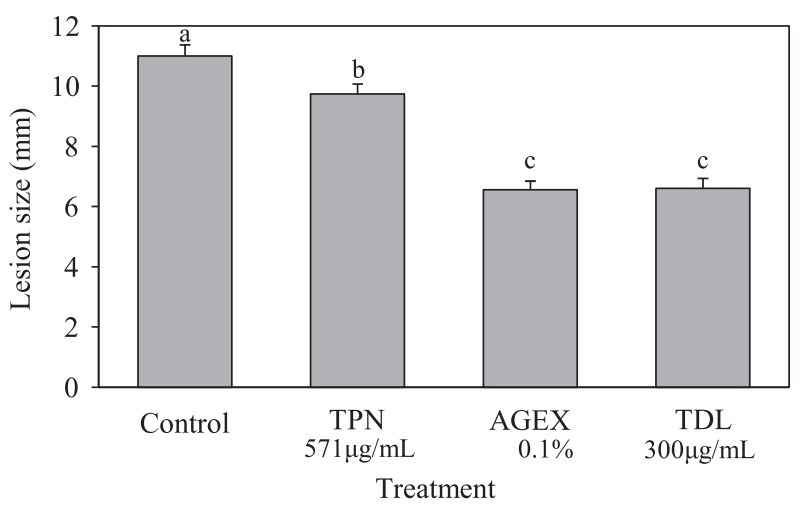

Fig. 2. Duration of induced resistance against gray blight in tea leaves treated with plant activators

Pestalotiopsis longiseta was inoculated 42 days after plant activator treatment. Lesion sizes were measured 14 days after inoculation. Values indicate the mean size of lesion $(n=32)$ and vertical bars represent standard error. Different letters denote significant differences at the $1 \%$ level by Tukey's test.

\section{Effect of PA on mycelial growth of Cts and PI}

The antifungal activities of the tested PAs were assessed by the mycelial growth of Cts and Pl on PA-supplemented Czapek's agar plates as shown in Table 2. The mean mycelial growths of Cts with PBZ, PDJ and TDL were 14.8, 33.4 and $14.0 \mathrm{~mm}$, respectively. PBZ and TDL inhibited mycelia growth of Cts. The mycelial growths of Pl with PBZ, PDJ and TDL were 43.8, 25.4 and 34.5 $\mathrm{mm}$, respectively. These three tested chemicals suppressed mycelial growth of Pl. On the other hand, AGEX, a yeast extract preparation, enhanced mycelial growth of $\mathrm{Pl}$ and Cts on Czapeck medium; therefore, AGEX doesn't have an antifungal activity.

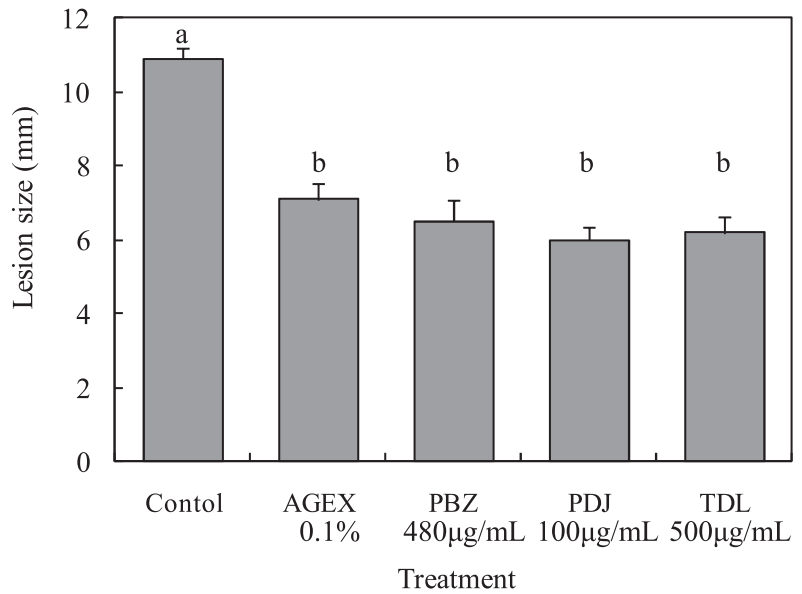

Fig. 3. Suppression of gray blight lesion development on upper leaves of plant activator-treated leaves

Pestalotiopsis longiseta was inoculated on the upper leaves of plant activator-treated lops 2 days after the treatment. Lesion sizes were measured 14 days after inoculation. Values indicate mean lesion size $(n=16)$ and bars represent standard error. Different letters denote significant differences at the $1 \%$ level by Tukey's test.

\section{Control efficacy of PA against tea fungal diseases}

We used AGEX, PBZ and TDL to examine the control of anthracnose and gray blight in the tea field. We did not use PDJ for the field trial because PDJ-induced disease resistance was less effective against Pl inoculation when the mature tea leaves were treated with PA under field conditions. The number of infected leaves was counted and the control efficacy of each PA was determined. The number of Cts-infected leaves was decreased by pretreatment with TDL, PBZ and AGEX (Table 3). However, the control efficacy of PAs against Cts was lower than that of TPN. In the case of control efficacy of 
PAs against $\mathrm{Pl}$, the number of $\mathrm{Pl}$-infected leaves also decreased with PA treatment, but their control efficacy was lower than that of TPN (Table 3).

\section{Effect of PA on growth of tea shoots and chemical constituents}

The effects of PAs on the growth of tea shoots were estimated 3 weeks after PA treatment. The length, weight and leaf number of tea shoots did not change after PA treatment (Table 4). The contents of total nitrogen, total free amino acids, neutral detergent fiber, tannin, and caffeine in PA-treated leaves were determined. There were no significant differences in each tea component in PAtreated leaves compared to the untreated control (Table 4).

Table 2. Effect of plant activators on mycelial growth of C. theae-sinensis and P. longiseta

\begin{tabular}{lcc}
\hline \multirow{2}{*}{ Treatment } & \multicolumn{2}{c}{ Mycelial size (mm) } \\
\cline { 2 - 3 } & Cts & Pl \\
\hline Control & $38.8 \mathrm{a}$ & $62.9 \mathrm{a}$ \\
AGEX $0.2 \%$ & $64.1 \mathrm{~b}$ & $85.0 \mathrm{~b}$ \\
PBZ $480 \mu \mathrm{g} / \mathrm{mL}$ & $14.8 \mathrm{c}$ & $43.8 \mathrm{c}$ \\
PDJ $100 \mu \mathrm{g} / \mathrm{mL}$ & $33.4 \mathrm{a}$ & $25.4 \mathrm{~d}$ \\
TDL $300 \mu \mathrm{g} / \mathrm{mL}$ & $14.0 \mathrm{c}$ & $34.5 \mathrm{e}$ \\
\hline
\end{tabular}

1) Mycelial growth was estimated 14 days after inoculation. Different letters denote significant differences at the $1 \%$ level by Tukey's test.

\section{Discussion}

There are many reports on induced disease resistance in crop and vegetable $1,3,4,18,20,24$. Induced resistance in fruit trees by ASM and SAR in annual plants have been studied ${ }^{2,7,8,11,19,34}$. However, induced disease resistance in tea plants and the PA activities of PBZ and TDL on woody plants have not been studied. Here, we estimated the PA activities of AGEX, PBZ, PDJ, and TDL on tea plants by wound-inoculation assays and field trials.

Wound-inoculation assays revealed that the application of AGEX, PBZ, PDJ, and TDL induces disease resistance in tea plants against $\mathrm{Cts}$ and $\mathrm{Pl}$ infections. Suppressions in $\mathrm{Cts}$ and $\mathrm{Pl}$ lesion development were observed in tea leaves treated with PAs, 30 days after PA treatment. In another experiment, spraying PAs onto emerging tea shoots induced disease resistance in tea leaves, 42 days after PA treatment. These results suggest that PAs induces long-lasting disease resistance both in mature leaves and emerging shoots of tea plants under field conditions. The induction of systemic disease resistance by PAs was confirmed by laboratory experiments. Lesion development in gray blight was suppressed in the upper leaves of PA-treated lops that were not directly treated with PAs. These results suggest that AGEX, PBZ, PDJ, and TDL induced systemic disease resistance in tea plants. Mycelial growths of Cts and Pl were observed on PA-supplemented Czapek's agar plates. In the tested concentrations, the mycelial growth of both the fungi was not completely inhibited by PAs, especially AGEX. The mycelial growths of Cts and Pl on AGEX additive plates were more than that on the control plate. These results suggested that AGEX, PBZ, PDJ, and TDL act as PAs on tea plants.

Table 3. Control efficacy of plant activators on anthracnose and gray blight in a tea field

\begin{tabular}{|c|c|c|c|c|}
\hline \multirow[b]{2}{*}{ Treatment } & \multicolumn{2}{|c|}{ Athracnose ${ }^{1)}$} & \multicolumn{2}{|c|}{ Gray blight ${ }^{2}$} \\
\hline & $\begin{array}{l}\text { Infected leaves } \\
\text { per } 1 \mathrm{~m}^{2}\end{array}$ & $\begin{array}{l}\text { Control } \\
\text { efficacy }\end{array}$ & $\begin{array}{l}\text { Infected leaves } \\
\text { per } 1 \mathrm{~m}^{2}\end{array}$ & $\begin{array}{l}\text { Control } \\
\text { efficacy }\end{array}$ \\
\hline Control & $893 \mathrm{a}^{3)}$ & - & $129 \mathrm{a}$ & - \\
\hline AGEX $0.1 \%$ & $446 \mathrm{~b}$ & 50.1 & $44 \mathrm{~b}$ & 65.7 \\
\hline $\mathrm{PBZ} 480 \mu \mathrm{g} / \mathrm{mL}$ & $373 \mathrm{~b}$ & 58.2 & $47 \mathrm{~b}$ & 63.7 \\
\hline TDL $500 \mu \mathrm{g} / \mathrm{mL}$ & 394 b & 55.9 & $39 \mathrm{~b}$ & 69.8 \\
\hline TPN $571 \mu \mathrm{g} / \mathrm{mL}$ & $202 \mathrm{c}$ & 77.4 & $11 \mathrm{c}$ & 91.5 \\
\hline \multicolumn{5}{|c|}{$\begin{array}{l}\text { 1) C. theae-sinensis was inoculated } 3 \text { days after PA treatment at the opening of the first tea leaf of the } \\
\text { tea shoot. } \\
\text { 2) P. longiseta was inoculated after the tea trees were cut and plant activators were treated } 4 \mathrm{~h} \text { after } \\
\text { inoculation. }\end{array}$} \\
\hline
\end{tabular}


The control efficacies of AGEX, PBZ and TDL on anthracnose and gray blight were determined by field trials. The number of Cts- and Pl-infected leaves was suppressed by AGEX, PBZ and TDL. However, the control efficacy of these three PAs was lower than that of TPN. The foliar application of PAs on tea plants induces disease resistance, but does not completely protect the tea plants against fungal infection. The timing, frequency and application methods of PAs should be studied to improve their control efficacy in the future.

SAR and ISR are regulated by different signaling pathways in plants and are induced by different types of pathogens and substances ${ }^{1,4,24}$. In Chinese cabbage, ASM induced resistance to anthracnose but ASM enhanced the severity of Alternaria leaf spot ${ }^{14}$. In contrast, methyl jasmonate and ethephon induced resistance to Alternaria leaf spot but not to anthracnose ${ }^{14}$. These results suggest that the selection of an appropriate PA is important for the control of specific plant diseases. Obara et al. ${ }^{15}$ reported that AGEX induces ethylene-dependent disease resistance that differs from SAR. In the present study, AGEX and PDJ induced long-lasting and systemic disease resistance in tea, like PBZ and TDL. Yoshida et al. ${ }^{29,30}$ reported that the wounding of tea leaves or methyl jasmonate induced an accumulation of proteinase inhibitor; however, the accumulation of proteinase inhibitor was inhibited by SA. In contrast, wounding, methyl jasmonate and SA induced the activation of phenylalanine ammonia-lyase (PAL) in tea plants ${ }^{30}$. PAL is associated with catechin biosynthesis ${ }^{10}$ and the defense reaction of tea plants against fungal infection ${ }^{31}$. These results suggest that AGEX and TDL might induce basic and com- mon self-defense mechanisms in tea plants; therefore, AGEX and TDL induce a similar level of disease resistance in tea plants. Further studies on the details of the defense mechanisms induced by AGEX and TDL are required to elucidate the induced disease-resistance mechanism in tea plants. AGEX induces disease resistance in tea plants in the same way as the synthetic PA of PBZ and TDL. AGEX is a preparation of baker's yeast extract and does not have fungicidal activity. These unique features of AGEX are very interesting and can be used for the development of a novel disease-resistant inducer from a natural product.

We analyzed the effect of PA-application on the growth and chemical composition of tea shoots. In some cases, PA exhibited plant toxicity with the induction of disease resistance ${ }^{13}$. Fumagalli et al. ${ }^{6}$ reported that ASM field application to grape increased anthocyanin content. These results indicate the possibility that PA application to tea plants could induce a negative effect on tea shoot growth or an increase in some polyphenolic compounds. In this study, PA did not affect growth or the chemical composition of tea shoots (Table 4). These results suggest that PAs induce disease resistance in tea without a negative effect on tea production. Further studies are required to examine the residual concentration of PAs in green tea products and the effect of PA application on green tea quality in tea plants.

PA induces systemic and long-lasting disease resistance in tea plants. The control efficacy of PA on anthracnose and gray blight is lower than that of TPN; however, the experimental results on the suppression of tea diseases by PA gave an insight into the development of new dis-

Table 4. Growth and chemical composition of tea shoots 3 weeks after the plant activator treatment

\begin{tabular}{|c|c|c|c|c|c|c|c|c|}
\hline \multirow[b]{2}{*}{ Treatment } & \multicolumn{3}{|c|}{ Shoot growth ${ }^{1)}$} & \multicolumn{5}{|c|}{ Chemical composition ( $\%$ of dry leaf weight $)^{2)}$} \\
\hline & $\begin{array}{l}\text { Shoot } \\
\text { weight } \\
\text { (g) }\end{array}$ & $\begin{array}{l}\text { Shoot } \\
\text { length } \\
(\mathrm{cm})\end{array}$ & $\begin{array}{l}\text { Number of } \\
\text { expanded } \\
\text { leaves }\end{array}$ & $\begin{array}{l}\text { Total } \\
\text { nitrogen }\end{array}$ & $\begin{array}{l}\text { Free } \\
\text { amino } \\
\text { acids }\end{array}$ & $\begin{array}{l}\text { Neutral } \\
\text { detergent } \\
\text { fiber }\end{array}$ & Tannin & Caffeine \\
\hline Control & 0.51 & 4.3 & 3.5 & 2.77 & 0.93 & 27.6 & 14.3 & 2.0 \\
\hline AGEX $0.1 \%$ & 0.55 & 4.8 & 3.7 & 2.77 & 0.83 & 27.5 & 14.5 & 2.2 \\
\hline $\mathrm{PBZ} 480 \mu \mathrm{g} / \mathrm{mL}$ & 0.49 & 4.9 & 3.8 & 2.73 & 0.93 & 28.1 & 14.3 & 2.0 \\
\hline PDJ $100 \mu \mathrm{g} / \mathrm{mL}$ & 0.54 & 4.7 & 3.7 & 2.77 & 0.87 & 27.9 & 14.0 & 2.0 \\
\hline $\mathrm{TDL} 500 \mu \mathrm{g} / \mathrm{mL}$ & 0.50 & 4.6 & 3.6 & 2.77 & 0.87 & 27.5 & 14.5 & 2.1 \\
\hline Tukey's test $(\mathrm{p}<0.01)$ & $\mathrm{ns}^{3)}$ & $\mathrm{ns}$ & $\mathrm{ns}$ & $\mathrm{ns}$ & $\mathrm{ns}$ & $\mathrm{ns}$ & $\mathrm{ns}$ & $\mathrm{ns}$ \\
\hline
\end{tabular}

Plant activators were sprayed on tea shoots of the first leaf development stage for the third tea crop.

1) Values are means of 40 tea shoots.

2) Values are means of chemical constituents from three replications.

3) Not significant. 
ease control methods for tea production. The duration of PA-induced disease resistance is enough to cover one tea crop season; therefore, dual disease control systems against anthracnose and gray blight might be developed by analyzing the details of the disease resistance mechanisms induced by PAs.

\section{Acknowledgments}

This study was conducted under the research project "Development of new biorational techniques for sustainable agriculture (2004-2009)" funded by the Ministry of Agriculture, Forestry and Fisheries in Japan.

\section{References}

1. Arie, T. \& Nakashita, H. (2007) Plant activator. Shokubutsu boeki (Plant Protection), 61, 531-536 [In Japanese].

2. Brisset, M, N. et. al (2000) Acibenzolar-S-methyl induces the accumulation of defense-related enzymes in apple and protects from fire blight. Eur. J. Plant Pathol., 106, 529536.

3. Cohen, Y., Gisi, U. \& Niderman, T. (1993) Local and systemic protection of Phytophthora infestans induced in potato and tomato plants by jasmonic acid and jasmonic methyl ester. Phytopathology, 83, 1054-1062.

4. Dong, X. (1998) SA, JA, ethylene and disease resistance in plants. Cur., Opin. Plant. Biol., 1, 316-323.

5. Faize, M. et al. (2008) Gene expression during acibenzolarS-methyl-Induced priming for potentiated responses to Venturia nashicola in Japanese pear. J. Phytopathol., 157, 137-144.

6. Fumagalli, F. et al. (2006) From field to health: A simple way to increase nutraceutical content of grape as shown by NO-dependent vascular relaxation. J. Agric. Food. Chem., 54, 5344-5349.

7. Hassan, M. A. E. \& Buchenauer, H. (2007) Induction of resistance to fire blight in apple by acibenzolar-S-methyl and DL-3-aminobutyric acid. J. P. Dis. and Protect., 114, 151158.

8. Ishii, H. et al. (1999) Induced resistance of acibenzolar-Smethyl (CGA 245704) to cucumber and Japanese pear diseases. Eur. J. Plant Pathol., 105, 77-85.

9. Iwai, T. et al. (2006) Contribution of ethylene biosynthesis for resistance to blast fungus infection in young rice plants. Plant Physiol., 142, 1202-1215.

10. Iwasa, K. (1976) Physiological aspects of catechin biosynthesis in tea plants. JARQ, 10, 89-93.

11. Kimberly, A. S. et al. (2002) Effect of treating apple trees with acibenzolar-S-methyl on fire blight and expression of pathogenesis-related protein genes. Plant Dis., 86, 785790 .

12. Koshiyama, M. et al. (2006) Development of a new plant regulator, prohydrojasmon. Shokubutsu no seichochosetsu (Reg. Plant Growth Develop.), 41, 24-33 [In Japanese].

13. Mandal, B. et al. (2008) Biological and molecular analysis of the acibenzolar-S-methyl-induced systemic acquired resistance in flue-cured tobacco against tomato spotted wilt virus. Phytopathology, 98, 196-204.

14. Narusaka, Y. et al. (2008) The effects of plant activators on leaf spot and anthracnose of Chinese cabbage. Nippon noyaku gakkaishi (J. Pestic. Sci.), 32, 196-200 [In Japanese with English summary].

15. Obara, N. et al. (2007) Mechanism of PR gene expression by treatment of tobacco leaves with yeast extract (AGREVO EX). Nippon shokubutsu byorigaku kaiho (Jpn. J. Phyotopathol.), 73, 94-101 [In Japanese with English summary].

16. Oniki, M., Hamaya, E. \& Ando, Y. (1985) The actual note of resistant strains of the tea anthracnose fungus Gloeosporium theae-sinensis against benzimidazole fungicides in Japan. Chagyo kenkyu hokoku (Tea Research Journal), 61, 7-11 [In Japanese with English summary].

17. Oniki, M., Narisawa, N. \& Ando, Y. (1986) Incidence of strains of the tea gray blight fungi Pestalotia longiseta and $P$. theae resistant to benzimidazol fungicides in Japan. Chagyo kenkyu hokoku (Tea Research Journal), 64, 29-33 [In Japanese with English summary].

18. Oostendorp, M. et al. (2001) Induced disease resistance in plants by chemical. Eur. J. Plant Pathol., 107, 19-28.

19. Qui, X. et al. (2004) Identification and expression analysis of BTH induced genes in papaya. Physiol. Mol. Plant Pathol., 65, 21-30.

20. Ryal, J. A. et al. (1996) Systemic acquired resistance. Plant Cell, 8, 1809-1819.

21. Staswick, P. E. et al. (1998) Jasmonate signaling mutants of Arabidopsis are susceptible to the soil fungus Pythium irregulare. Plant $J ., 15,747-754$.

22. Takeda, Y. (2002) Genetic analysis of tea gray blight resistance in tea plants. JARQ, 36, $143-150$.

23. Thomma, B. P. H. J. et al. (1998) Separate jasmonate-dependent and salicylate-dependent defense response pathway in Arabidopsis are essential for resistance to distinct microbial pathogens. Proc. Natl. Acad. Sci. USA, 95, 15107-15111.

24. Vallad, G. E. \& Goodman, R. M. (2004) Systemic acquired resistance and induced systemic resistance in conventional agriculture. Crop Sci., 44, 1920-1934.

25. Vijayan, P. et al. (1998) A role for jasmonate in pathogen defense of Arabidopsis. Proc. Natl. Acad. Sci. USA, 95, 7209-7214.

26. Yanase, Y. \& Takeda, Y. (1987) Method for testing the resistance to tea gray blight caused by Pestalotia longiseta Spegazzini in tea breeding. Yasai chagyo shikenjyo hokoku (Bull. Natl. Res. Inst. Veg. Orn. Plants and Tea), B 1, 1-9 [In Japanese with English summary].

27. Yasuda, M. et al. (2004) Tianidil, a novel class of activators of systemic acquired resistance, induces defense gene expression and disease resistance in tobacco. J. Pestic. Sci., 29, 46-49.

28. Yasuda, M. et al. (2006) Thiadiazole carboxylic acid moiety of tiadinil, SV-03, induces systemic acquired resistance in tobacco without salicylic acid accumulation. J. Pestic. Sci., 31, 329-334.

29. Yoshida, K. et al. (2000a) Systemic wound responses in tea plant. Nippon sakumotsugakkai tokaishibu kaiho (Rep. Tokai Br. Crop Sci. Soc. Japan), 130, 9-11 [In Japanese with English summary].

30. Yoshida, K. et al. (2000b) Effect of chemical treatments on 
wound responses in tea leaves. Nippon sakumotsugakkai tokaishibu kaiho (Rep. Tokai Br. Crop Sci. Soc. Japan), 130, 13-16 [In Japanese with English summary].

31. Yoshida, K. et al. (2000c) Characterization of disease resistance related proteins in tea leaves. Nippon sakumotsugakkai tokaishibu kaiho (Rep. Tokai Br. Crop Sci. Soc. Japan), 130, 17-21 [In Japanese with English summary].

32. Yoshida, K. \& Takeda, Y. (2006) Evaluation of anthracnose resistance among tea genetic resources by wound-inoculation assay. $J A R Q, \mathbf{4 0}, 379-386$.

33. Yoshioka, K. et al. (2001) Probenazole induces systemic acquired resistance in Arabidopsis with a novel type of action. Plant J., 25, 149-157.

34. Zhu, Y. J. et al. (2003) Systemic acquired resistance induced by BTH in papaya. Physiol. Plant Mol. Pathol., 63, 237-248. 